\title{
Genetic Association between Dopamine Transporter Protein Alleles and Cocaine-Induced Paranoia
}

\author{
Joel Gelernter, M.D., Henry R. Kranzler, M.D., Sally L. Satel, M.D., and Peter A. Rao, M.D.
}

\begin{abstract}
Paranoia in the context of cocaine abuse is common and potentially dangerous. Several lines of evidence suggest that this phenomenon may be related to function of the dopamine transporter protein (DAT). DAT is the site of presynaptic reuptake of dopamine, an event that terminates its synaptic activity. The gene coding for dopamine transporter protein (DAT1) contains a variable number of tandem repeats (VNTR) polymorphism in the $3^{\prime}$ untranslated region that can be typed by the polymerase chain reaction ( $P C R$ ) (Vandenbergh et al. 1992). Although this is not a coding region polymorphism, it is close to the coding region and could plausibly be in linkage disequilibrium with a mutation in the gene. Cocaine blocks the dopamine transporter and increases synaptic availability of dopamine. We examined DAT alleles in 58 white and 45 black cocaine users in
\end{abstract}

KEY WORDS: Cocaine abuse; Dopamine transporter protein (DAT); Paranoia

Cocaine dependence is a major public health problem in the United States. Certain sequelae of cocaine abuse are dangerous and unpredictable. Genetic differences in the makeup of individuals who abuse cocaine may

From the Departments of Psychiatry, Yale University School of Medicine and West Haven VA Medical Center, West Haven, Connecticut, and the Department of Psychiatry, University of Connecticut School of Medicine, Farmington, Connecticut.

Address correspondence to: Joel Gelernter, Psychiatry 116A, West Haven VA Medical Center, 950 Campbell Avenue, West Haven, CT 06516

Received February 4, 1994; revised April 21, 1994; accepted June 10, 1994. order to test only two hypotheses: (1) Is there an allelic association between DAT and cocaine dependence? and

(2) Is there an allelic association between DAT and cocaine-induced paranoia?

We did not demonstrate an allelic association with cocaine dependence. However, within the white sample, $D A T$ genotype was associated with cocaine-induced paranoia (allele frequency for allele $9=.16$ for those without paranoid experiences versus .35 for those with, $\chi^{2}=3.9[2 \times 2$ table $\left.], p<.05\right)$. There was no significant difference for the same measure in the black sample. Certain DAT genotypes may therefore predispose to paranoia in the context of cocaine use in white populations. We caution that these results require independent replication. [Neuropsychopharmacology 11:195-200, 1994] underlie some of these differences in susceptibility to particular drug effects.

Psychotic symptoms (such as paranoia) are sometimes attributed to an excess of synaptic dopamine activity. There could be many molecular mechanisms eventually resulting in excess synaptic dopamine, including, for example, abnormalities in dopamine metabolism or in dopamine receptors. The dopamine transporter protein (also called the dopamine reuptake site; genetic locus DAT1) is another possible site of such a lesion. Abnormal dopamine transport could easily result in abnormal levels of synaptic dopamine. Cocaine is thought to exert many of its actions through blocking dopamine reuptake by binding the DAT (Ritz et al. 1987), and it also regulates DAT mRNA expression (Xia et al. 1992). Genetic variation in DAT could alter its interaction with cocaine. 
When administered intravenously, cocaine has been shown to precipitate transient paranoid states (Sherer et al. 1988), an effect that may be dopaminergically mediated (Post 1975; Sherer 1988). In patients seeking treatment for cocaine dependence, 53 to $68 \%$ report having experienced paranoia (i.e., fears unrelated to real environmental threats such as apprehension by police) associated with cocaine use (Brady et al. 1991; Satel et al. 1991).

Several studies have also provided evidence for a link between cocaine use and violent behavior, with paranoid symptoms often prominent in the clinical presentation. Manschreck and colleagues (1988) reported that in the course of a cocaine epidemic in the Bahamas, violent behavior was present in $55 \%$ of psychotic cocaine abusers and in $36 \%$ of nonpsychotic cocaine abusers. Paranoid ideation was present in all psychotic patients who were violent. Brody (1990) described 37 patients with violent or aggressive behavior associated with cocaine intoxication, which represented greater than $4 \%$ of all cocaine-related visits to the medical emergency department of a busy urban hospital over a twoyear period. In $62 \%$ of these patients, violent behavior occurred in association with cocaine-induced delirium, paranoia, or other alterations in mental status.

Behavioral disturbances such as those that occur in the context of cocaine intoxication, though common, are not universal. Staley and colleagues (1993) sought to identify alterations in the dopamine transporter that might contribute to the behavioral effects of cocaine. Using quantitative in vitro autoradiography, these investigators found elevated [125I]RTI-55 binding in striatum in a group of individuals who had died from cocaine overdose but who had not manifested behavioral abnormalities. In contrast, there was no upregulation observed in striatum in individuals who died following a cocaine-induced delirium. These differences were thought to suggest a diminished capacity for dopamine reuptake following cocaine exposure in individuals susceptible to delirium.

Because of the clinical importance of the problem of cocaine-induced paranoia and the evidence connecting this symptom to the dopamine transporter, we studied genetic variation of DAT1 in a series of cocainedependent individuals. We hypothesized that (a) there might be a difference in allele frequency between cocaine-dependent individuals and other populations due to DAT alleles varying susceptibility; and (b) that different DAT alleles might alter susceptibility to cocaine-induced paranoia.

The gene coding for dopamine transporter protein has been mapped to distal chromosome $5 p$ by physical methods (Vandenbergh et al. 1992), a location confirmed by us by linkage analysis (unpublished data). It contains a variable number of tandem repeats (VNTR) polymorphism in the $3^{\prime}$ untranslated region that can be typed by the polymerase chain reaction (PCR) (Vandenbergh et al. 1992). Although this is not a coding region polymorphism, it is close to the coding region and could plausibly be in linkage disequilibrium with a mutation in the gene. (However, it is not in linkage disequilibrium with a more $5^{\prime}$ RFLP polymorphism; Vandenbergh et al. 1992).

\section{SUBJECTS AND METHODS}

Subjects were 103 individual meeting DSM-III-R diagnostic criteria for cocaine dependence (see below). Age at time of assessment was $34.1 \pm 6.4$ (SD) years. There were 58 white and 45 black subjects. Cocaine-induced paranoia appears to be a condition to which white and black individuals are equally vulnerable (Brady et al. 1991; Satel et al. 1991). Six of the subjects were female.

\section{Diagnosis}

All subjects were seeking treatment for drug dependence. All met DSM-III-R criteria for a lifetime diagnosis of cocaine dependence (American Psychiatric Association 1987). That diagnosis was made in $85 \%$ of the subjects using a computerized version of the Diagnostic Interview Schedule for DSM-III-R (C-DISR; Blouin et al. 1989; Levitan et al. 1991). Among the remainder of the subjects, the diagnosis was made using the Structural Clinical Interview for DSM-IIIR (SCID; Spitzer et al. 1992). Subjects were excluded if they had a clinical diagnosis of schizophrenia, another psychotic disorder, or an organic mental syndrome, or if during the period of their heaviest cocaine use they were also using intravenous heroin.

A history of cocaine-induced paranoia was assessed using a modified version (H. Pettinati, personal communication) of the Cocaine Experience Questionnaire (CEQ; Satel et al. 1991). This instrument assesses methods and patterns of cocaine use, the context and nature of paranoid symptoms associated with use, and whether or not such symptoms accompanied the use of other substances. Family history of functional psychotic symptoms was elicited during administration of the questionnaire. The subjects were carefully instructed to distinguish adaptive hypervigilance or anxiety in high-risk situations (e.g., making drug deals, passing through housing projects, engaging in illicit activities) from completely irrational beliefs (e.g., the perception that the police were standing on the window ledge outside the subject's tenth floor apartment). This questionnaire was administered by a rater and typically took 20 minutes for a subject who did not report drugrelated paranoid experiences and 30 to 45 minutes for a subject who did. After an explanation, subjects were asked, "Have you ever had a paranoid experience?" 


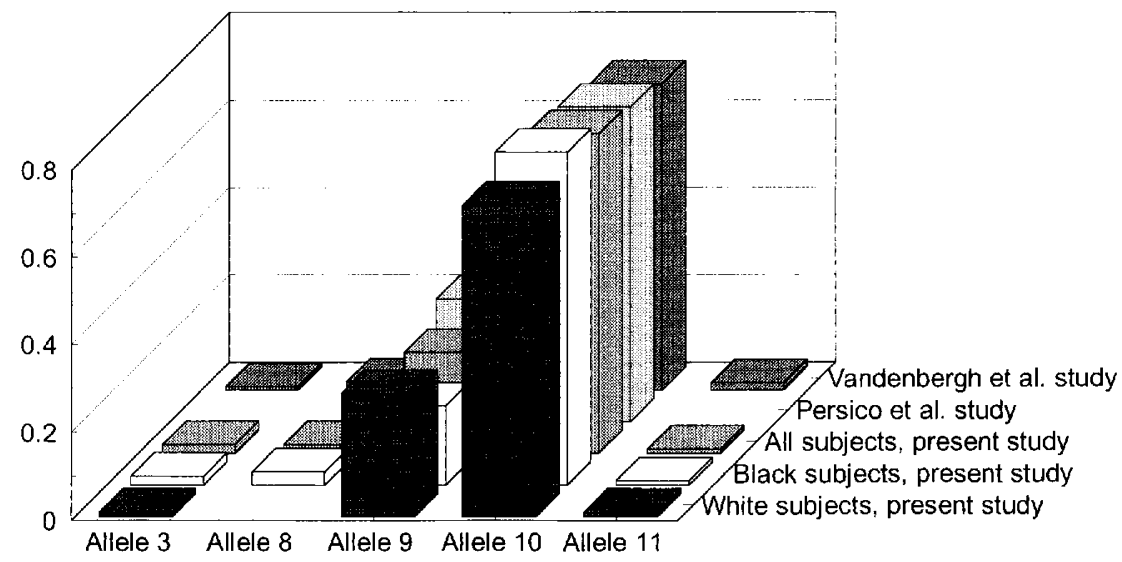

Figure 1. Observations of allele frequencies at DAT1 for our sample; white subjects, black subjects, and the entire sample. For comparison, we give data from Persico et al. 1993 (white substance abusers) and Vandenbergh et al. 1992 (white controls). Vandenbergh et al. 1992 also observed the following rare alleles and frequencies: allele 5 (.008); allele 7 (.012).
Those who reported having had such an experience were then asked, "Have you had a paranoid experience when you were using cocaine?" Only subjects who answered affirmatively to both of these questions were considered to be positive for cocaine-induced paranoia. The interrater reliability of this distinction was assessed in 19 subjects. This yielded a concordance rate of $89.5 \%$ and a value of kappa, a statistic that accounts for chanceexpected agreement (Fleiss 1981) of 0.77 , which is considered excellent (Cicchetti and Sparrows 1981). The test-retest reliability of this distinction was assessed in 24 subjects, with a mean ( \pm SD) of $100.2( \pm 78.6$ ) days and a range of 11 to 238 days between assessments. This yielded a concordance rate of $79.2 \%$ and a value of kappa of 0.57 , which is considered fair.

\section{Genotyping}

The polymerase chain reaction (PCR) was used to amplify a 200 to 520 base segment of the $3^{\prime}$ untranslated region of the DAT gene (size depending on the number of 40-base repeats), as described by Vandenbergh et al. (1992). Alleles were assigned on the basis of the size of the PCR products generated for reactions using genomic DNA from cocaine abusing individuals as template. The numeric designation of each allele refers to the number of repeats it contains.

\section{Analyses}

We used genetic association data to address two hypotheses: (1) Is there an allelic association between DAT and cocaine dependence? and (2) Is there an allelic association between DAT and cocaine-induced paranoia?

Statistical significance was tested using standard $\chi^{2}$ tests for $2 \times 2$ tables, comparing frequencies of either allele 9 or 10 (by far the two most common alleles) with the sum of the other alleles. We divided the sample only once, by presence or absence of cocaineinduced paranoia, to minimize the potential problem of repeated statistical tests.

\section{RESULTS}

Overall, the allele frequencies observed at the DAT 3' VNTR were similar to those observed both in a population sample of Caucasians (Vandenbergh et al. 1992) and in a polysubstance abusing sample (Persico et al. 1993) (Fig. 1). There was no significant difference in allele frequencies between our black and white subjects, which is consistent with the observation of Vandenbergh et al. (1992); however, paranoia data were analyzed separately by race, because of the possibility that there are population differences in DAT allele frequencies, or in linkage disequilibrium between the DAT VNTR that we assessed and a putative mutation of physiological effect.

In the white subjects, there was a significant difference between those with and those without paranoia in the frequency of the 9 allele (allele frequencies 0.35

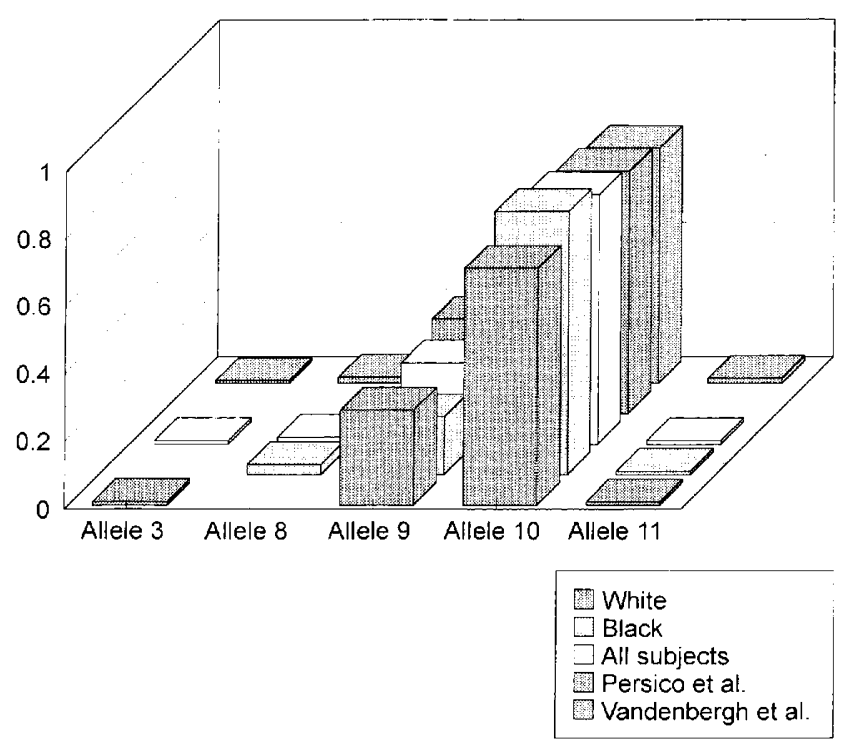

Figure 2. Allele frequencies in our sample for white subjects according to presence or absence of cocaine-induced paranoia $(n=58)(p=.047$ for allele 9$)$. 
Table 1. DAT1 Alleles and Cocaine-Induced Paranoia

\begin{tabular}{lcc}
\hline & Allele 9 & Other Alleles \\
\hline White subjects $(n=58)$ & & 37 \\
Paranoia $(-)(n=22)$ & 75 & 47 \\
Paranoia $(+)(n=36)$ & & 43 \\
Chi square $=3.9, p=0.047$ & 7 & 31 \\
Black subjects $(n=45)$ & 9 & \\
Paranoia $(-)(n=25)$ & & \\
Paranoia $(+)(n=20)$ & & \\
Chi square $=0.59$, not significant (NS) & 14 & \\
All subjects $(n=103)$ & 34 & Other Alleles \\
Paranoia $(-)(n=47)$ & & \\
Paranoia $(+)(n=56)$ & & \\
Chi square $=6.0, p=0.014$ & Allele $\mathbf{1 0}$ & \\
& & \\
White subjects $(n=58)$ & 36 & 11 \\
Paranoia $(-)(n=22)$ & 46 & \\
Paranoia $(+)(n=36)$ & & \\
Chi square $=3.4, p=0.065$ & & \\
Black subjects $(n=45)$ & 39 & \\
Paranoia $(-)(n=25)$ & 29 & \\
Paranoia $(+)(n=20)$ & & \\
Chi square $=0.13$, NS & & \\
All subjects $(n=103)$ & 75 & \\
Paranoia $(-)(n=47)$ & & \\
Paranoia $(+)(n=56)$ & & \\
Chi square $=3.7, p=0.057$ & & \\
\hline
\end{tabular}

and 0.16 , respectively; $\chi^{2}=3.9 ; p=.047$ ) (Figure 2). There was also a difference in frequency of the 9 allele between those subjects with and those without paranoia in the entire sample (i.e., black and white subjects combined; allele frequencies 0.30 and 0.15 for paranoia $(+)$ and $\left.(-) ; \chi^{2}=6.0 ; p=.014\right)$. There were no statistically significant associations between allele frequency and cocaine-induced paranoia in the black subjects. (Results are summarized in Tables 1 and 2.)

\section{DISCUSSION}

These results demonstrate a statistically significant relationship between DAT alleles and susceptibility to cocaine-induced paranoia in white cocaine abusers. This suggests that vulnerability to cocaine-induced paranoia may be influenced by allelic variation at the DAT gene.

To minimize risk of a false positive, we tested only two hypotheses. These hypotheses were defined a priori on the basis of pathophysiological data relating the protein product of the DAT gene and the action of cocaine in vivo. This was demonstrated in a racially (although not ethnically) homogeneous subsample. However, genetic association studies are inherently susceptible to false positive results (Gelernter et al. 1993).

We would like to call attention to several limitations of this study that are common to many studies of this type. First, we do not know if the polymorphism we studied has any direct physiological effect. Given its location $3^{\prime}$ to the gene, it seems rather unlikely that it

Table 2. Summary of Allele Frequencies

\begin{tabular}{llllll}
\hline Group $(\boldsymbol{n})$ & Allele 3 & Allele 8 & Allele 9 & Allele 10 & Allele 11 \\
\hline White (58) & $0.01(1)$ & 0 & $0.28(32)$ & $0.71(82)$ & $0.01(1)$ \\
Black (45) & $0.02(2)$ & $0.03(3)$ & $0.18(16)$ & $0.76(68)$ & $0.01(1)$ \\
White paranoia (-) (22) & 0 & 0 & $0.16(7)$ & $0.82(36)$ & $0.02(1)$ \\
White paranoia (+) (36) & $0.01(1)$ & 0 & $0.35(25)$ & $0.64(46)$ & 0 \\
Black paranoia (-) (25) & $0.02(2)$ & $0.06(3)$ & $0.14(7)$ & $0.78(39)$ & 0 \\
Black paranoia (+) (20) & $0.03(1)$ & 0 & $0.23(9)$ & $0.73(29)$ & $0.03(1)$ \\
All subjects (103) & $0.02(3)$ & $0.02(3)$ & $0.23(48)$ & $0.73(150)$ & $0.01(2)$ \\
All paranoia (+) (56) & $0.02(2)$ & 0 & $0.30(34)$ & $0.67(75)$ & $0.01(1)$ \\
All paranoia (-) (47) & $0.01(1)$ & $0.03(3)$ & $0.15(14)$ & $0.80(75)$ & $0.01(1)$ \\
\hline
\end{tabular}


would have such an effect. The plausibility of these results carrying physiological meaning would therefore depend primarily on whether or not this polymorphism is in linkage disequilibrium with an expressed mutation. This is less unlikely; because the polymorphism we assessed is physically adjacent to the gene, if there were such a mutation (especially if it were relatively $3^{\prime}$ within the gene), it could well be in linkage disequilibrium with a nearby polymorphic marker. This could be demonstrated by mutation screening (Gejman and Gelernter 1993). Second, our sample size of 58 white subjects leaves us at risk for a chance finding based on random variation. Therefore, acceptance of these results should depend on independent replication and eventual demonstration of an expressed mutation.

There was also a statistically significant difference in frequency of the 9 allele at DAT1 among those subjects with versus without cocaine-induced paranoia in the entire (black plus white) sample that was greater than the difference observed in the white sample alone. This could reflect either a real difference in the black sample too small to detect in that racially homogenous population alone, or an artifact based on (presently unknown) differences in allele frequencies between black and white populations. It will be necessary to study allele frequencies at this locus in a larger black population in order to determine which of these explanations is correct.

The reliability with which subjects were identified as having experienced cocaine-induced paranoia was excellent when rated by different interviewers. The reliability of the distinction appeared to be lower, however, when evaluated over an extended period. Given that reliability sets an upper limit for validity, this would suggest that the degree of association observed between DAT allele frequencies and cocaine-induced paranoia may be underestimated in this study. Refinement of this distinction, with a concomitant increase in the reliability with which it is made, would be important in the context of subsequent efforts to validate this association.

If these results were replicated, it would suggest that variation in the dopamine transporter protein could play a role in determining interindividual variation to cocaine-induced paranoia. It would then be interesting to determine if DAT variants could contribute to susceptibility to other psychotic symptomatology.

\section{ACKNOWLEDGMENTS}

The authors wish to thank Laura Green (technical assistance), Claudia Aguero, Despina Beazoglou, Kitzia Skipsey (data collection assistance), and Paula Butterfield (data management and analysis). The work was supported in part by funds from the U.S. Department of Veterans Affairs (USVA National
Centers for PTSD and Schizophrenia Research, the VA-Yale Alcoholism Research Center, and the VA Medical Research Program [Merit Review grants]), NIMH grant K20-MH00931 to JG, NIDA grants DA04060 and DA05592, and NIAAA grant K20-AA00143 to HK, and a VA Research Training Fellowship in Psychiatry (PR).

\section{REFERENCES}

Blouin AG, Perez EL, Blouin JH (1988): Computerized administration of the Diagnostic Interview Schedule. Psychiatry Res 23:335-344

Brady KT, Lydiard RB, Malcolm R, Ballenger JC (1991): Cocaine induced psychosis. J Clin Psychiatry 52:509-511

Brody SL (1990): Violence associated with acute cocaine use in patients admitted to a medical emergency department. In Drugs and Violence: Causes, Correlates, and Consequences. Rockville, MD: National Institute on Drug Abuse, DHHS publication number (ADM) 90-1721, pp. 44-58.

Cicchetti DV, Sparrows SS (1981): Developing criteria for establishing interrater reliability of specific items: Applications to the assessment of adaptive behavior. Am J Ment Defic 86:127-137

Fleiss JL (1981): Statistical Methods for Rates and Proportions, 2nd ed, New York, Wiley

Gejman PV, Gelernter J (1993): Mutational and analysis of candidate genes in psychiatric disorders. Am J Med Genet 48:184-191

Gelernter J, Goldman D, and Risch N (1993): The A1 allele at the $D_{2}$ dopamine receptor gene and alcoholism: $A$ reappraisal. JAMA 269:1673-1677

Levitan RD, Blouin AG, Navarro JR, Hill J (1991): Validity of the computerized DIS for diagnosing psychiatric inpatients. Can J Psychiatry 36:728-731

Manschreck TC, Laughery JA, Weinstein CC, Allen D, Humblestone B, Neville M, Podlewski H, Mitra N (1988): Characteristics of freebase cocaine psychosis. Yale J Biol Med 61:115-122

Persico AM, Vandenbergh DJ, Smith SS, Uhl GR (1993): Dopamine transporter gene polymorphisms are not associated with substance abuse. Biol Psychiatry 34:265-267

Post R (1975): Cocaine psychosis: A continuum model. Am J Psychiatry 132:225-231

Ritz MC, Lamb RJ, Goldberg SR, Kuhar MJ (1987): Cocaine receptors on dopamine transporters are related to selfadministration of cocaine. Science 237:1219-1223

Satel SL, Southwick S, Gawin F (1991): Clinical features of cocaine-induced paranoia. Am J Psychiatry 148:495-499

Sherer MA (1988): Intravenous cocaine: Psychiatric effects, biological mechanisms. Biol Psychiatry 24:865-885

Sherer MA, Kumor KM, Cone EJ, Jaffe JH (1988): Suspiciousness induced by four-hour intravenous infusions of cocaine. Arch Gen Psychiatry 45:673-677

Spitzer RL, Williams JBW, Gibbon M, First MB (1992): The Structured Clinical interview for DSM-III-R: 1 . History, rationale and description. Arch Gen Psychiatry 49:624629 
Staley J, Basile M, Wetli CV, Hearn WL, Flynn DD, Ruttenber AJ, Mash DC (1993): Differential regulation of the dopamine transporter in cocaine overdose deaths. Presented at the Annual Meeting of the College on Problems of Drug Dependence, Toronto, Canada, June 12-17, 1993

Vandenbergh DJ, Persico AM, Hawkins AL, Griffin CA, Li X, Jabs EW, Uhl GR (1992): Human dopamine transporter gene maps to chromosome 5p15.3 and displays a VNTR. Genomics 14:1104-1106

Xia Y, Goebel DJ, Kapatos G, Bannon MJ (1992): Quantitation of rat dopamine transporter mRNA: Effects of cocaine treatment and withdrawal. J Neurochemistry 59: 1179-1182 